\title{
3D Virtual World Retrieval Based on Ontology and Content
}

\author{
Elmustapha Ait Lmaati ${ }^{1}$, Abdellah Ait Ouahman ${ }^{1}$, Mohammed Najib Kaddioui ${ }^{1} \&$ Mohammed Sadgal $^{1}$ \\ ${ }^{1}$ Cadi Ayyad University, UCA, Marrakesh, Morocco \\ Correspondence: Elmustapha Ait Lmaati, Cadi Ayyad University, UCA, Marakesh, Morocco. E-mail: \\ lmaatimustapha@yahoo.fr
}

Received: January 20, 2014

Accepted: February 17, 2014 Online Published: March 20, 2014

doi:10.5539/cis.v7n2p36

URL: http://dx.doi.org/10.5539/cis.v7n2p36

\begin{abstract}
On the Web and on informatics systems, 3D virtual worlds used become big both in number and in size. Therefore, we propose in this paper a new method for retrieving 3D virtual worlds based on the semantics and the content. Firstly, we propose a new classified database of 3D virtual worlds given in VRML format. To achieve the semantic method, we construct an ontology that describes virtual worlds in various aspects including their contents (3D objects building a virtual world) and information about their contents (authors, file format, etc). This ontology is presented by OWL, the W3C recommended language. So as to extract desired 3D scenes from the proposed database we use the SPARQL query language. We propose finally a shape based method for searching desired virtual worlds by content in this database. This method is based on a new distance (metric) that define the similarity between virtual worlds. This method is evaluated using the recall vs. precision curves.
\end{abstract}

Keywords: ontology, SPARQL, 3D virtual world retrieval, OWL, semantic web

\section{Introduction}

With the development of software and 3D scanner that permit to build 3D objects, 3D virtual worlds including the 3D objects become more used around the web both in number and size. Those 3D virtual worlds are used in various domains like game, engineering, physical simulation etc. Considering this situation, finding desired 3D scenes from 3D virtual world database become difficult. The solution is to find new method for indexing and retrieving by semantic and by content those virtual worlds that contain sets of 3D objects.

Many researcher proposed yet search engines for 3D objects (Princeton search engine) (Informatics \& Telematics Institute search engine) (ENIC search engine). Those search engines give user the possibility to search 3D objects by examples or by text key words from databases. The content based method are based in general on shape and use deformable (Niccolucci \& D'Andrea, 2006) or non deformable 3D models (Chen et al., 2003; Ansary et al., 2007; Lmaati et al., 2009, 2010a, 2010b, 2010c). The text based methods are in general based on key words describing 3D object given recently in XML format.

With the development of the semantic Web also called the intelligent web or next generation web or Web 3.0., $\mathrm{RDF}$ (2004) is used to represent information and to exchange knowledge on the Web. OWL, the recommended web ontology language is used for describing things of the web. SPARQL (2008) language an official W3C recommendation is an RDF (2004) query language that is used to retrieve and manipulate data stored by ontology. All these semantic web tools are important to improve the semantic 3D virtual world retrieval.

The semantic based methods are proposed in the literature (Horrocks, 2008; Gong et al., 2011; Kassimi et al., 2012; Niccolucci et al., 2006; Xin-ying et al., 2008). These methods use in general ontologies so as to describe $3 \mathrm{D}$ models. In our work we propose to describe and to retrieve using an ontology not just 3D models, but virtual worlds that contain sets of 3D models and other concepts building virtual world like graphical environments (illumination, fog, etc.) and animation of 3D models (translation, rotation, etc).

Practically, a set of 3D objects are used for building a 3D virtual world. Therefore, in order to find desired 3D virtual worlds from a database, we proceed to find 3D virtual worlds that contain the set of 3D objects constituting the virtual world query. Matching two 3D virtual worlds problematic is solved by matching two sets of 3D objects. For this, we define the distance (metric) between two sets of 3D objects using distances between 3D objects.

In this paper, we propose in the section 2 a database of virtual words. This database is a set of VRML (2001) 
virtual words built by a set of 3D objects collected from Internet. The system overview is presented in section 3 . This retrieval system is decomposed into the on-line process and the off-line process. In section 4 , we propose a semantic based description using ontology of virtual worlds given in format VRML. Then we use in section 5 the SPARQL (2008) language to retrieve from our dataset desired virtual worlds in different level. For example "find the virtual worlds that contain red cars" or "find fog forest virtual worlds that have white horses". We give in section 6 our approach for retrieving 3D virtual worlds by shape content of the objects constituting the virtual world. This matching process is based on computing distances between objects of a pair of virtual worlds which is evaluated using recall vs. precision curve. At the end, we present the conclusion.

\section{The 3D Virtual World Database}

We provide 50 virtual worlds that use 3D objects collected from the Princeton shape benchmark (PSB) (Shilane, 2004) database and from internet. The used objects that build those virtual worlds are given by meshes or by grouping the graphical basic forms including cone, sphere, box, cylinder, etc. Those virtual worlds have different media type including graphic, audio, image, text, animated objects etc. The set of virtual worlds constituting the database are classified by semantic aspects into 10 classes.

All virtual worlds of our database are given in format VRML (Virtual Reality Modeling Language, 2001). VRML is the most useful and the standard on the Web. It is a scene description language that enables the construction of interactive, animated 3-D models and virtual worlds on Web pages.

\section{- The classification}

The proposed classification is based on the idea that if most objects of a virtual world belong to a unique domain, we consider this domain as the class including this virtual world. Figure 1 shows some virtual worlds of our database from different classes.

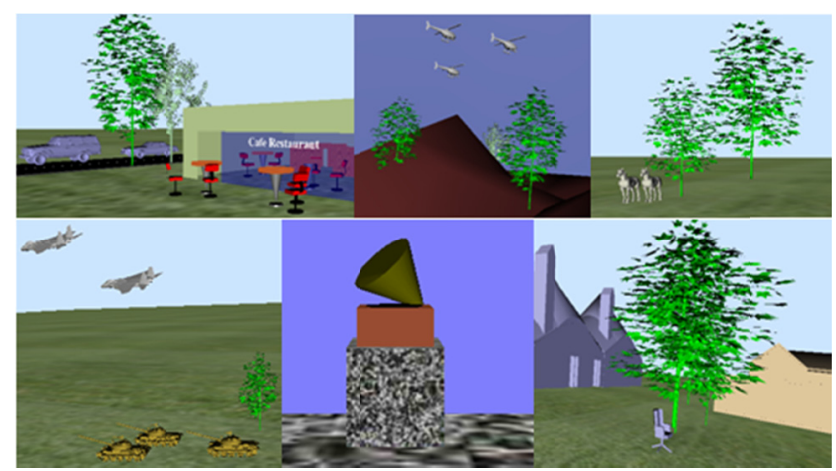

Figure 1.3D virtual worlds from our database

The class 1: this class is named 'restaurant'. It has 6 different virtual worlds. The used objects in this class are tables, building of the coffee, glasses, bottles, chairs, etc. that belong the domain "restaurant". The Figure 2 shows the thumbnails of the virtual worlds that constitute this class.

The class 2: this class is named 'road'. It contains 4 virtual worlds. The most used 3D objects to build those virtual worlds are cars, road, etc.

The class 3: this class is named 'war'. It contains 14 different $3 \mathrm{D}$ virtual worlds. The most used 3D objects to construct the virtual worlds of this class are plans (fighter-jet), tanks, etc.

The class 4: this class is named 'commercial plans-cars'. It contains 5 3D virtual worlds. The most used 3D objects to construct the virtual worlds of this class are commercial plans and cars.

The class 5: this class is named 'mountain'. It contains $33 \mathrm{D}$ virtual worlds. The most used objects to construct the virtual worlds of this class are trees, mountains, etc.

The class 6: this class is named 'Garden'. It contains 7 3D virtual worlds. The most used 3D objects to construct the virtual worlds of this class are trees, domestic animals, etc.

The class 7: this class is named 'building'. It contains $53 \mathrm{D}$ virtual worlds. The most used objects to construct the virtual worlds of this class are buildings, trees, etc.

The class 8: this class is named ' $3 \mathrm{D}$ scene with audio'. It contains 2 virtual worlds. The most used objects of this 
class are obtained by grouping some basic forms (box, cylinder, cone, etc) and music (audio file) at the backgrounds.

The class 9: this class is named 'Fogged 3D scene'. It contains trees and other 3D objects in 3D scenes with Fog.

The class 10: this class is named "Forest". The virtual worlds of this class contain trees, animals, etc.

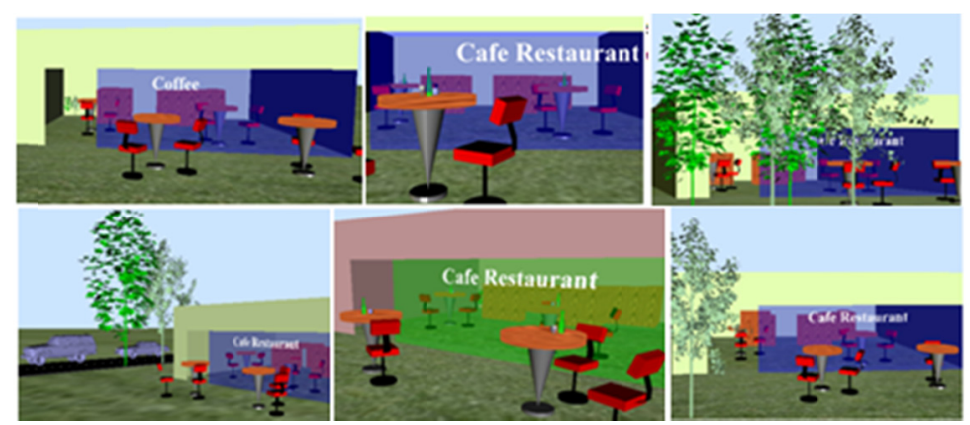

Figure 2. The class "restaurant" from our database

\section{System Overview}

The proposed system illustrated in Figure 3 is composed into two parts which are the off-line part and the on-line part. The on-line process permit for users to navigate in our 3D virtual world database, select a virtual world as a query, send it to the server and receive similar virtual worlds to the query. The returned virtual worlds using this query by example is based on shapes of objects belonging virtual worlds in our database. To achieve the matching process, the system computes distances between objects that belonging the virtual world query and objects that belonging virtual worlds in the database and computes the distance $\mathrm{D}$ (given in section 6) between the virtual world query and the virtual worlds in our database. By choosing semantic query, the user can also search desired virtual worlds basing on semantic concepts by using the text based interface. The extracted virtual worlds for SPARQL query are given in section 6 . In the off-line part, the system uses the proposed ontology that stores both the semantic information and the content based information (shape based descriptors) for virtual worlds in the proposed database. The shape based descriptors for 3D objects that belonging the virtual worlds in the database are generated using different methods known in the literature. Our experience uses the 3DCA: the 3D curve analysis descriptor given in reference (Lmaati et al., 2010c) for matching virtual worlds by content.

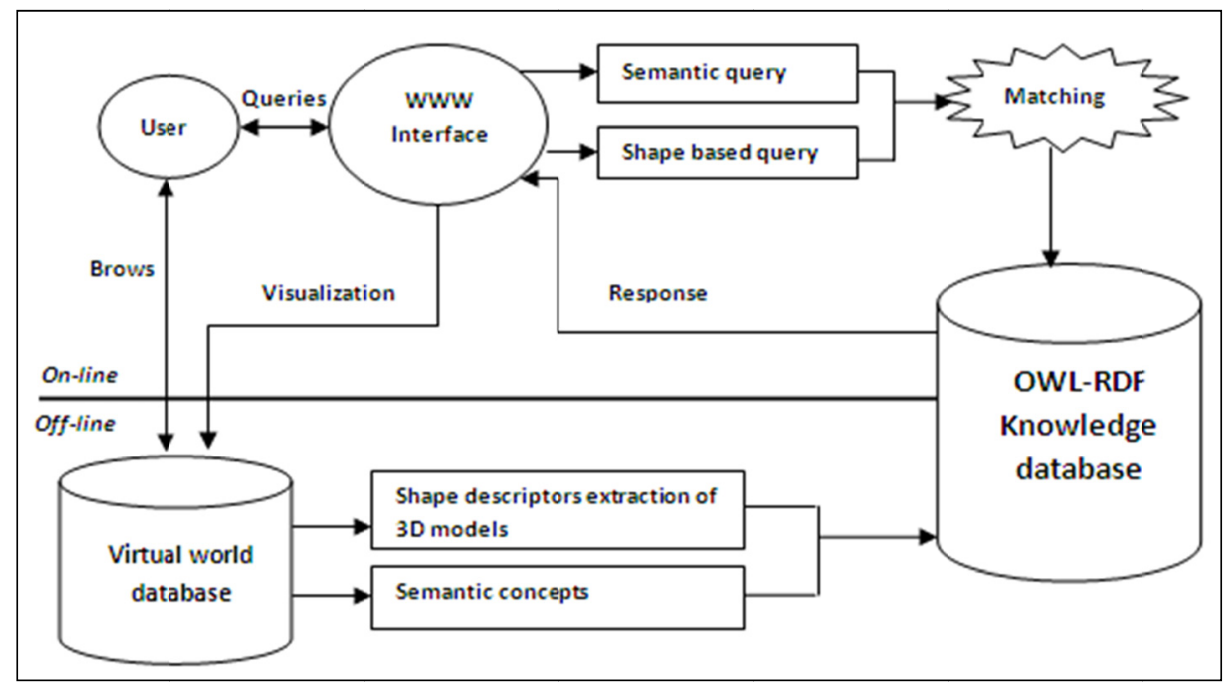

Figure 3. Architecture of the system 


\section{3D Virtual World Ontology}

\subsection{Ontology Structure}

We are developing the 3D virtual world ontology so as to give description regarding the semantic (author of the virtual world, size and type of files used to build it) and the media content of the virtual world including objects given by meshes, objects given by basic forms (box), images used as texture or for the backgrounds, text, video and audio and the environment like (fog or illumination). This ontology describes as possible the used components to build the virtual worlds so as to retrieve them better.

The virtual world ontology that we propose is developed within the Protégé (2013) editor. This permit to generate the OWL (2004) file based on RDF/XML representation of the ontology. The graph given in Figure 4 shows the classes and subclasses used to describe a 3D virtual world. Those classes are used to describe the virtual worlds which are subclass of the main class 'Thing'. The main class virtual_world 3D is a subclass of 'Thing', represent the virtual world description. The main class 'Object' present an abstraction of objects used to build the virtual world like 'table', 'tree', 'plane', etc. This class has subclasses: 1- 'Complex_Obj-mesh' that presents the objects of the virtual world given by meshes. 2- 'Group_Basic_Forms_Obj' that presents the objects obtained by grouping a set of basic geometric forms like box and cylinder. 3 - Box, 4-Cylinder, 5 - Cone, $6-$ Indexed_Face_Set, 7 - Sphere, 8 -HalfCone, 9 -Torus, 10 -HalfCylinder and 11-HalfSphere. The class 'Image' presents the images used to build the virtual world background or other. The class texture presents the image used for texturing objects used in the virtual world. The class text presents the object text used in the virtual world as shown in the Figure 2.

The classes: image, texture, text, and audio_BG are subclasses of the root class 'Thing'. They represent the abstraction of all media that can be part of a virtual world. The classes: Color, Illumination, Fog and Floor are subclasses of 'Thing'. They are used to describe the environment and the background of the virtual world.

The classes: Meta-Info_semantic, Creation_Date, Author, Names_dif-Language and StorageProperty are subclasses of 'Thing'. Those classes represent the metadata about the representation of semantic information about the virtual world and its objects.

The class Animation_Object is subclass of 'Thing', it describes the object animations used to build the virtual world.

The classes: Shape_Desc_3D, Speech_Ds, Image_Des_2D are subclasses of 'Thing' that permit to store the 3D shape descriptor, the speech descriptor and the image descriptor. The class shape_Des_3D has subclasses: Geometry_based, Feature_based, Graph_based as given by Tangelder et al. in reference (Tangelder \& Veltkamp, 2008). The Geometry_based has subclasses: Weighted_point_set, Deformation_based, Volumetric_error and View_based. The feature_based has subclasses spatial_map, global_features and local_features.

Since a variety of 3D- shape descriptors are proposed in the literature, we choose the Light Field (LF) (Chen et al., 2003), the 3D-DCT (Lmaati et al., 2010b) and the 3DCA (Lmaati et al., 2010c) as the 3D descriptors used by our ontology so as to store their components. The classes LFD, TD-DCT and ThreeDCA are subclasses of the class Global_features.

The image_Des_2D that permits to store the components of image descriptors has two subclasses: the Image_2D_Des_Color class and the Shape_Des_2D class.

In this section we give the different kinds of relationships between concepts for the proposed ontology as presented in Figure 6. A 3D virtual world presented by the class Virtual_world_3D can have the audio in the background and the text; those relations are given by the relationships has_Audio_BG and has_text respectively. The Virtual_world_3D has the Meta information presented by the class Meta-Info_semantic, it has also the storage property presented by the class StorageProperty; the graphics in the background (exp. image) description, environment (exp. fog) description and objects presented by the class Object. Those relations are presented by has_semanticDs, has_StorageProperty, has_GraphicsBG-env and has_Objects respectively. The Meta-Info_semantic is a class that has Author, Creation_Date and Names-dif_Languages; those relations are presented by has_Author, has_Creation_Date and has_Names respectively.

The class Object has color or texture, has storage property, has the Meta Information, can be animated and has a 3D shape description (example: ThreeDCA: 3DCA (Lmaati et al., 2010c)); those relationships are presented by has_Color, has_Texture, has_StorageProperty, has_semanticsDs, is_animatedBy, has_SD_3DCA respectively.

The class Graphics_Env_BG_Des has illumination, can have Floor, can have Images or Textures and can have a Fog environment. Those relationships are presented by has_Illumination, has_Floor, uses_Image, has_Texture 
and has_Fog. The Illumination, Fog, Text and the Floor use the color; this relationship is presented by uses_Color. The Floor can use Images or Textures; those relationships are presented by uses_Image and has_Texture respectively.

The class Image has storage property, has semantic Description (Meta-Info_Des), can have a 2D shape description and can have the 2D color image description. Those relationships are presented by: has_semanticDs, has_2D_Shape_Des, has_StorageProperty and has_Image_2D_Color_Des respectively. The Texture has semantic description and storage property; those relations are presented by has semanticsDs and has_StorageProperty. The audio_BG has it speech description (speech_Ds), storage property and semantic description; those relationships are presented by has_speech_Ds and has_StorageProperty and has_semanticsDs.

The class group_basic_Forms is a subclass of object. It presents the object that can be obtained by grouping the basic forms like Box, cylinder, Torus, etc.; this relationship is presented by has_basic_Form.

The class Animation_Object has the properties Translation, Rotation and Deformation. The class Author has properties Author-Af (the author affiliation), Author_Name (name of author) and Author_Bio (the author biography). The class Color has the property Color_Data (example blue). The class Creation_Date has the property C_Date of type date. The class Floor has Elvation_grid and Relief as data properties. The class Fog has visibility_Range as data property. The class Illumination has data properties Intensity, Ambient_Intensity, Directional-Light and Point-Light. The class Image_2D_Des_Color stores the components of the image descriptor. The class Shape_Des_2D stores the components of the 2D shape descriptor. The class Names_dif-Languages stores names in different languages as the Names_lab property. The class Box (subclass of Object) stores its size as components: sizeX, sizeY and sizeZ.

The class Cone (subclass of Object) stores the heightCone and bottomRadius as its data properties. The class Cylinder (subclass of Object) stores the heightCylinder and RadiusCylinder as its data properties. The class Sphere (subclass of Object) stores its radius as RaduisSphere as a data property. The class Indexed_Face_Set stores Coord-index, points and solid-face as data properties describing the set of faces. The class ThreeDCA stores the components of the 3D shape descriptor (example 3DCA (Lmaati et al., 2010c)) as a data property. The class SpeechDs stores the components of the speech descriptor as data type property. The StorageProperty class stores the storage property of a used file including its format (exp: vrml, jpg) and its size in Kilo octets. The class text stores the text string as a data property. The class Virtual_world_3D stores the Thumbnail of the virtual world file using its URI.

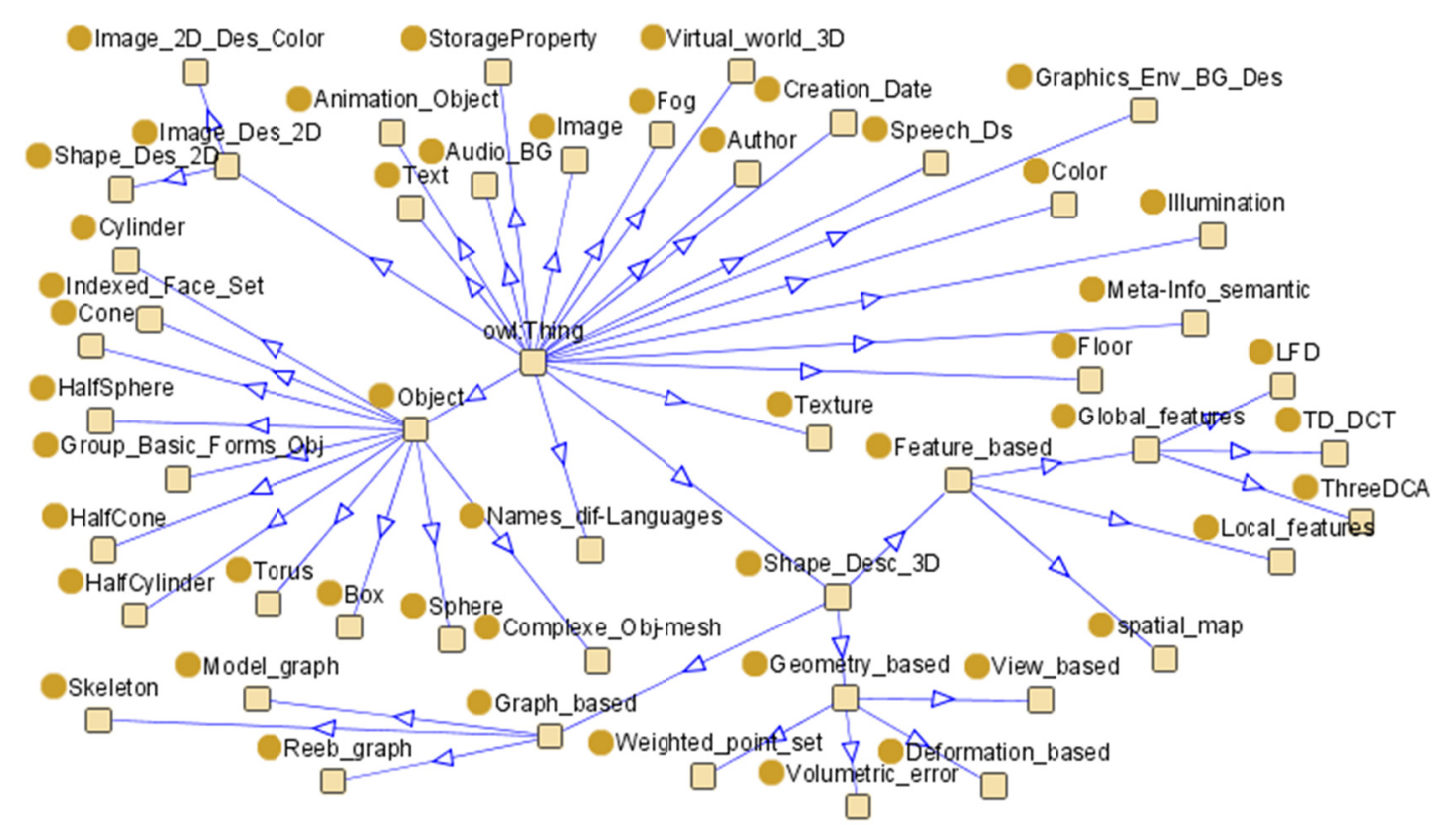

Figure 4. Classes and subclasses relationships of the proposed virtual world ontology 


\subsection{Annotation of the Virtual Worlds from the Database}

The virtual worlds stored in the database are annotated using protégé (Protégé, 2013). This process presented in Figure 5, shows the mapping of a virtual world to RDF/XML code stored via the ontology in an OWL (Ontology Web Language, 2004) file. For each virtual world in the database, we create a set of individuals representing things including the virtual worlds. Those things are given by the proposed ontology built in the precedent section presented by classes, subclasses, object properties and data properties.

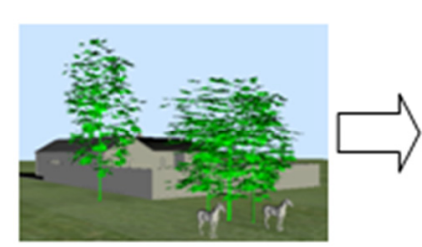

\section{RDF \\ Virtual World Annotation}

Figure 5. The virtual world annotation process

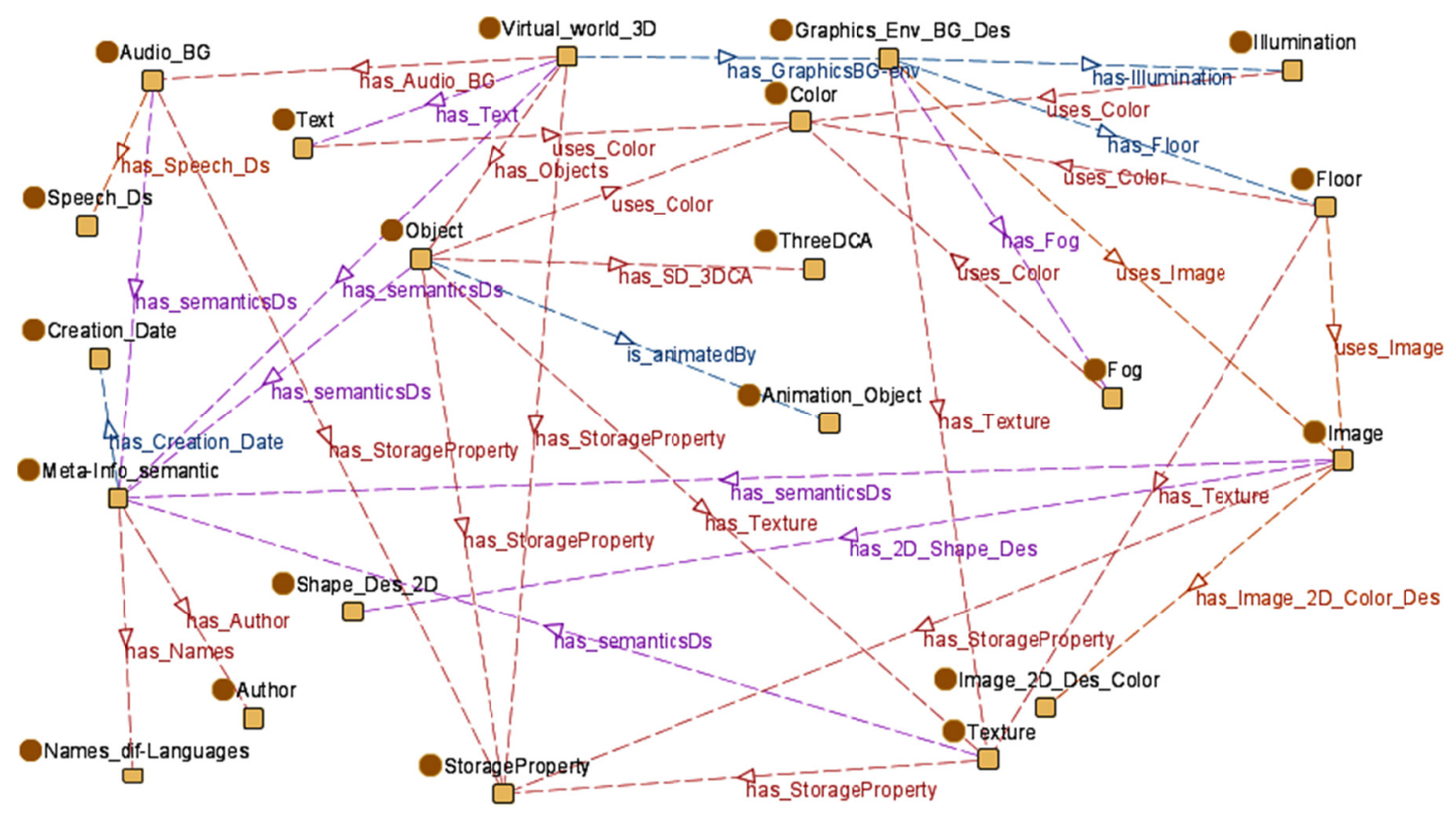

Figure 6. Concept relationships of the 3D virtual world ontology

For the example of virtual world given in the Figure 5, we create an instance of the class virtual_world 3D that we name as VWx, after we create the instances: Meta-Inf_semantic_VWx, storageProperty_VWx, Audio_BG_VWx and Text_VWx of the classes Meta-Inf_semantic, StorageProperty, Audio_BG and Text respectively. We create the instances horse1, horse2, building, tree1, tree2, tree3, tree4 as the instances of the class Object for the instance VWx. For the instance Meta-Inf_semantic_VWx, we create the instance Author_VWx and for Aurhor_VWx we store the string values of the properties Author_Name, Author_Affiliation and Author_Biography. For the instance horse1, we store the string value "white" as the value of an instance color_white of the class Color. This instance color_white is used as a value of the instance of the object property uses_Color. We denote that an instance can be used by more than one virtual world, object etc. for example: virtual worlds with the same authors or objects with the same colors.

\section{Semantic Based 3D Virtual World Retrieval Using SPARQL}

To achieve the semantic 3D virtual world retrieval, we are using the SPARQL (Protocol and RDF Query Language, 2008). This query language is recommended by W3C, used to extract the graph from an RDF file. SPARQL is able to gives response answer of complex query asked by user like (forest virtual worlds created on '23-12-2013' and that contain white horse?). The user query is transformed by the system to the SPARQL query. 
Using SPARQL, we show that we can extract desired virtual worlds from the database using query in different level. The level 1 retrieving virtual world with semantic and content properties; example: virtual worlds named "string" and created by an author.

The level 2 define description of objects in the virtual worlds; example: "retrieving virtual world containing objects with some properties". Using our ontological system we get those responses to the following SPARQL query. The Figure 7 gives the SPARQL query 1 that permits to retrieve the road 3D virtual worlds from our database and the relevant 3D virtual worlds from our database for this query.

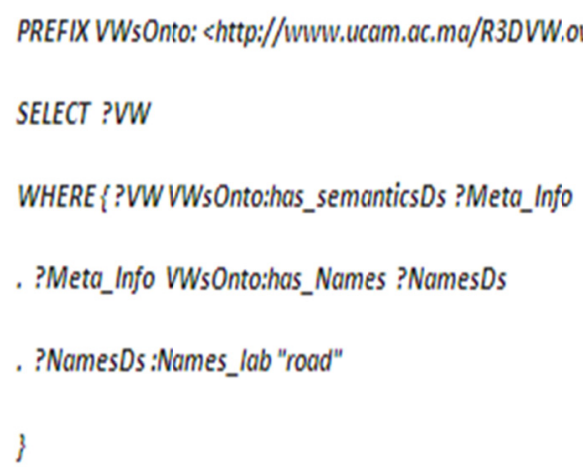

PREFIX VWSOnto: 〈http://Www.ucam.ac.ma/R3DVW.owl\#>

SELECT ?VW

WHERE\{?VWWW'Onto:has_semanticsDs ?Meta_Info

. ?Meta_Info WWsOnto:has_Names ?NamesDs

. ?NamesDs :Names_lab "road" \}

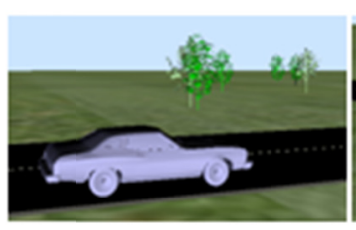

1: vw7

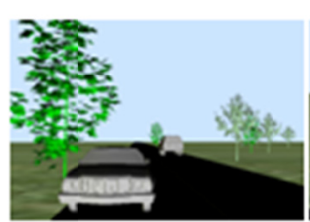

3: VW9

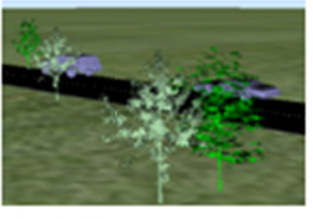

2: VWs

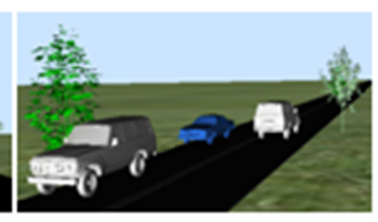

4: VW10

Figure 7. SPARQL query 1 retrieving road virtual worlds and the relevant virtual worlds for this query

The Figure 8 shows the SPARQL query 2 retrieving virtual worlds that contain an object "car" from the database. The extracted $3 \mathrm{D}$ virtual worlds from our database for this query contain a car.

PREFIX VWsOnto: <http://WWw.ucam.ac.ma/R3DVW.owl\#>

SELECT distinct ?VW

WHERE \{?VW VWsOnto:has_Objects ?Object

. ?Object VWsOnto:has_semanticsDs ?Meta_info

. ?Meta_Info VWsOnto:has_Names ?NamesDs

. ?NamesDs:Names_lab "car"

\}

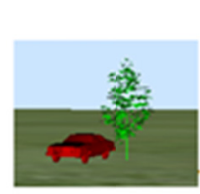

vis16

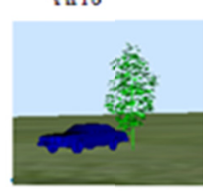

vw15

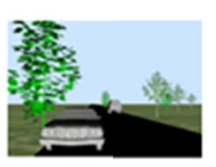

vw'9

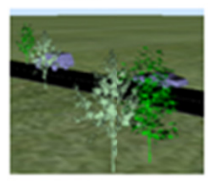

W's

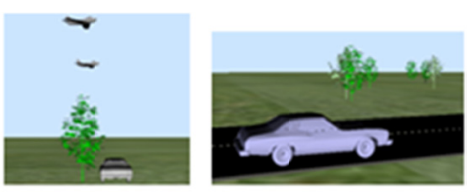

พw17

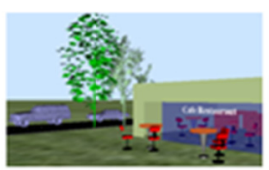

$\mathrm{w}^{2} 2$

1พ7

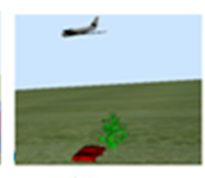

vw13

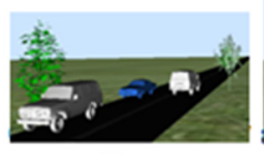

ทพ10

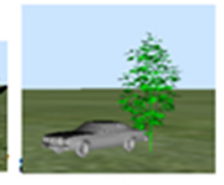

IW14

Figure 8. SPARQL query 2 retrieving the virtual worlds that contain a car and the returned virtual worlds from our database for this query

The query given in Figure 9 permits to retrieve "The virtual worlds that contain white horse". This SPARQL query 3 retrieves from our database the virtual worlds containing objects with properties. The figure shows that all the returned virtual worlds contain a horse. 


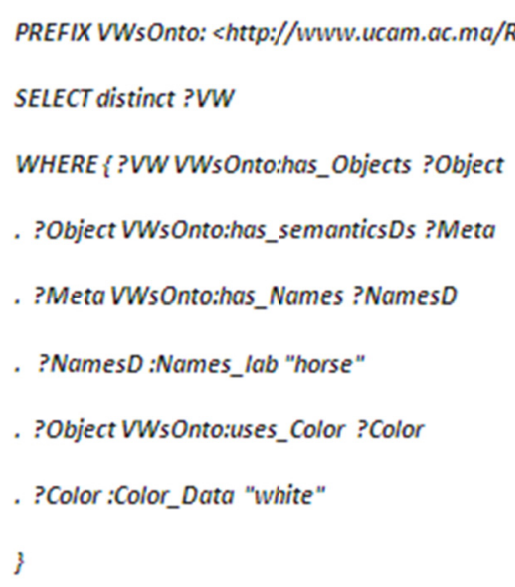

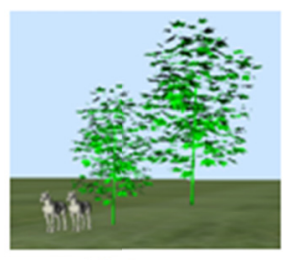

VW35

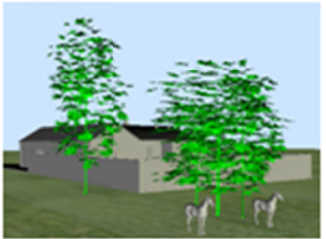

IT'41

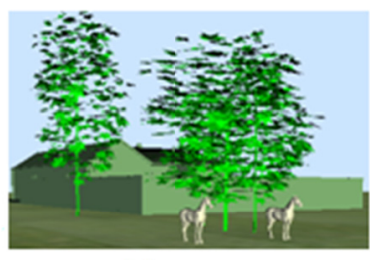

VW'42

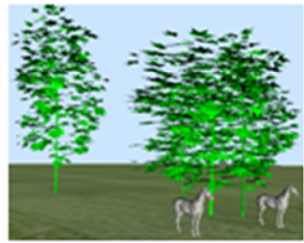

YW/37

Figure 9. SPARQL query 3 retrieving the virtual worlds that contain white horse and the returned virtual worlds from the database for this query

The SPARQL query 4 given in Figure 10 retrieves "The author names of fog forest virtual worlds". This query retrieves both the visual and semantic information of virtual worlds. This query returns the virtual world VW47 given in Figure 9 and the author name "E.A.Lmaati".

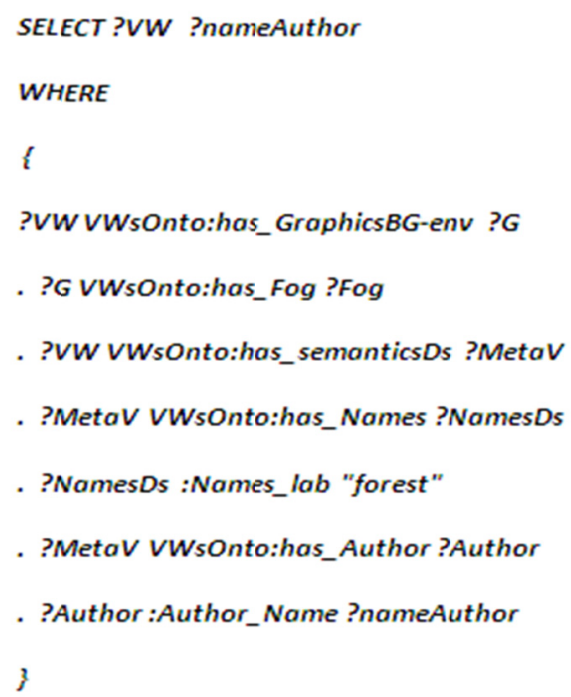

Figure 10. SPARQL query 4 retrieving the author names of the fog forest virtual worlds and the returned virtual world VW47

The given SPARQL queries show that using the proposed ontology that stores both the semantic information and the visual contents, we can retrieve using SPARQL desired 3D virtual worlds from our database.

\section{Content Based Retrieval for 3D Virtual Worlds}

Many works are achieved concerning the 3D object retrieval. Those methods are categorized into many classes. The first category concerns the non deformable 3D shapes (Chen et al., 2003; Ansary et al., 2007; Lmaati et al., 2009, 2010a, 2010b, 2010c). The second category concerns the deformable 3D shapes (Tabia et al., 2011). Those methods are used for matching only 3D shapes given in general by meshes. In this paper we propose a method for matching $3 \mathrm{D}$ virtual worlds that contain automatically $3 \mathrm{D}$ objects and extracting similarity between $3 \mathrm{D}$ virtual worlds. 
Since a virtual world contains a set of 3D objects, we denote that two virtual worlds are similar if for each object in the first virtual world there exist a similar object in the second virtual world and vice versa. Figure 11 shows two similar virtual worlds (VW:a and VW:b) since:

- For the object 1 in the virtual world VW:a there exist a similar plane (object 5) in the virtual world VW:b.

- For the object 2 in the virtual world VW:a there exist a similar plane (object 5) in the virtual world VW:b.

- For the object 3 in the virtual world VW:a there exist a similar tree (object 6 ) in the virtual world VW:b.

- For the object 4 in the virtual world VW:a there exist a similar tree (object 6 ) in the virtual world VW:b.

- For the object 5 in the virtual world VW:b there exist a similar plane (object 1) in the virtual world VW:a.

- For the object 6 in the virtual world VW:b there exist a similar tree (object 3 ) in the virtual world VW:a.

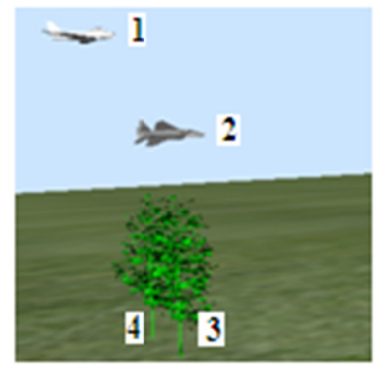

VW:a

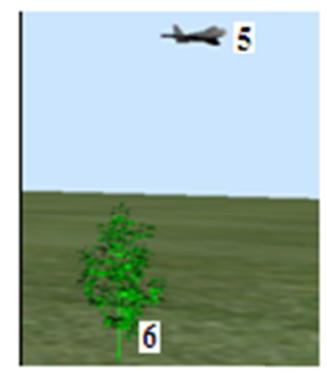

VW:b

Figure 11. Two similar virtual worlds

The Figure 12 shows two not similar (dissimilar) virtual worlds because there are not similar objects that belong the two virtual worlds VW:a and VW:c.

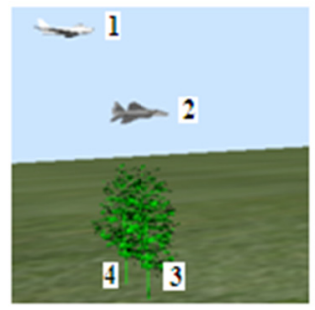

vw:a

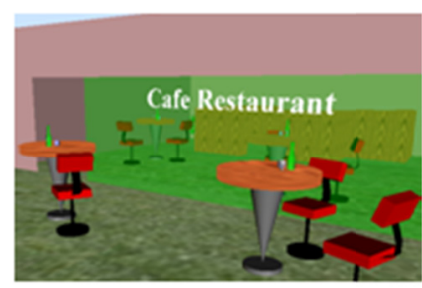

VW:c

Figure 12. Two dissimilar virtual worlds

The proposed ontology stores the descriptors for each 3D object belonging to the virtual world as a set of real value components. This descriptor is generated by aligning 3D models into canonical position using the continuous principal component analysis (CPCA) (Lmaati et al., 2010c), extracting 3D closed curve representing 3D model. Two descriptors the area and the dot product descriptors are extracted from the reconstructed 3D closed curve in order to define the 3D curve analysis descriptor (3DCA) (Lmaati et al., 2010c). Figure 6 shows that an object has a 3D shape descriptor 3DCA (has_SD_3DCA relationship) presented by the class ThreeDCA.

In order to compute similarity between two virtual worlds, we propose to compute the dissimilarity between two sets of 3D objects basing on the dissimilarity between objects from the virtual world query and other virtual worlds from the database. We consider that two virtual worlds are similar if the objects of the first virtual world are similar to the objects of the second virtual world and vice versa.

Let give a query VWq from the database. We consider that this virtual world query contains $\mathrm{n} 3 \mathrm{D}$ objects. For matching this virtual world to another virtual world named VW in the database, we compute the dissimilarity using the distance $\mathrm{D}$ given by the following formula: 


$$
\mathrm{D}\left(\mathrm{VW}_{\mathrm{q}}, \mathrm{VW}\right)=\frac{1}{\mathrm{r}} \sum_{k=1}^{r} \min _{i=1 . . p}\left(d\left(\boldsymbol{O}_{k}^{q}, \boldsymbol{O}_{i}\right)\right)+\frac{1}{\mathrm{p}} \sum_{i=1}^{p} \min _{k=1 . . r}\left(d\left(\boldsymbol{O}_{k}^{q}, \boldsymbol{O}_{i}\right)\right)
$$

Where $O_{k}^{q}$ is an object from the virtual world query and $O_{i}$ is an object from a virtual world from the database; $r$ is the number of the objects of the virtual world query and $p$ is the number of the objects of the virtual world VW from the database. The distance $\mathrm{d}$ between two 3D objects is calculated using a 3D shape descriptor given in the taxonomy added to the proposed ontology. In our experiment result we use the descriptor named 3DCA: 3D curve analysis feature vectors proposed by Lmaati in reference (Lmaati et al., 2010c).

It is evident that $\mathrm{D}$ is a distance between virtual worlds since $d$ is a distance between objects. $\mathrm{D}$ verify: the non-negativity, the symmetry, the reflexivity and the triangular inequality properties given in the following formulas:

i) $\quad \mathrm{D}(\mathrm{VW} 1, \mathrm{VW} 2)>=0$ for each VW1, VW2 in the database.

ii) $\quad \mathrm{D}(\mathrm{VW} 1, \mathrm{VW} 1)=0$ for each VW1 in the database.

iii) $\quad \mathrm{D}(\mathrm{VW} 1, \mathrm{VW} 2)=\mathrm{D}(\mathrm{VW} 2, \mathrm{VW} 1)$ for each VW1, VW2 in the database.

iv) $\quad \mathrm{D}(\mathrm{VW} 1, \mathrm{VW} 3)<=\mathrm{D}(\mathrm{VW} 1, \mathrm{VW})+\mathrm{D}(\mathrm{VW} 2$, VW3) for each VW1, VW2, VW3 in the database.

We denote that the proposed distance $\mathrm{D}$ performs well and more important to retrieve virtual worlds since it reflect the idea that two virtual worlds are similar if for each object in the virtual world query there exist an object similar to it in the second virtual world and vice versa, experimentally, The Figure 14 shows a virtual world query and the extracted virtual worlds using the distance D from our database. This figure shows that the extracted virtual worlds are similar to the query. The proposed Distance D can be used also for 2D image that contains more than one 2D shape.

We are using the recall vs. precision curves (Van Rijsbergen, 1979) so as to evaluate our method for retrieving by content 3D virtual worlds based on the distance D. In general the virtual worlds in the database are classified into p classes. For a class $C$ with $n$ 3D virtual worlds and for a given query $Q$ in a class $C$, let Rk be the number of correctly retrieved Virtual worlds among the $\mathrm{K}$ best matches. The recall is defined as a ratio of relevant virtual worlds $\mathrm{Rk}$ to $(\mathrm{n}-1)$, and the precision is the ratio of the relevant results and returned results $\mathrm{K}$. The Figure 13 shows the recall vs. precision plots using the distance D. It shows that this distance D performs well using our $3 \mathrm{D}$ virtual world database since the values of precision are high.

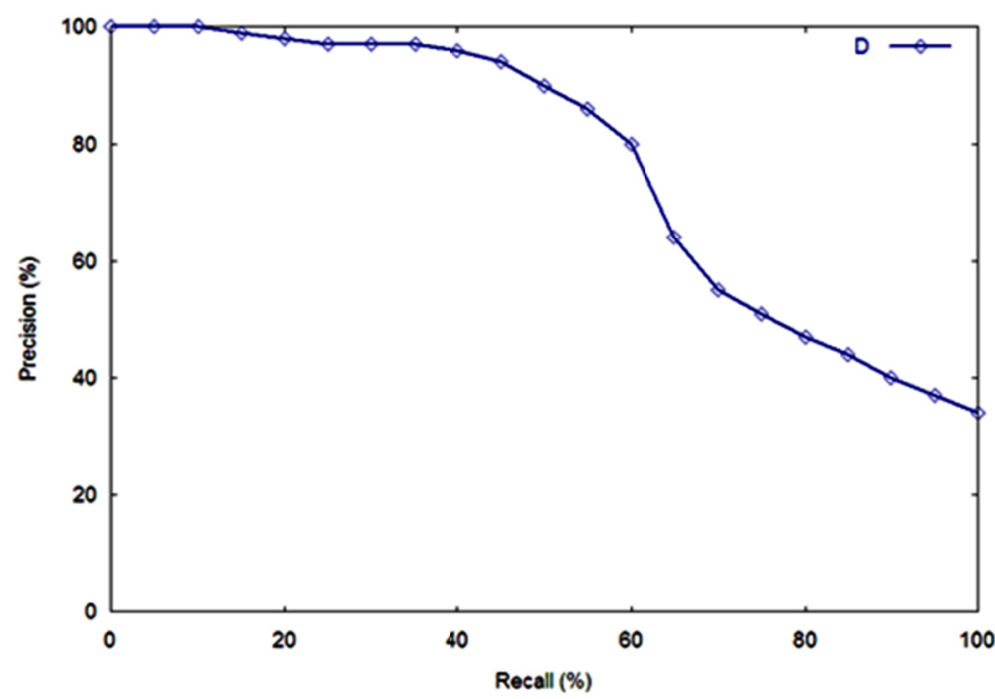

Figure 13. Recall vs. precision plot using the distance D for the 'war' class from our database 


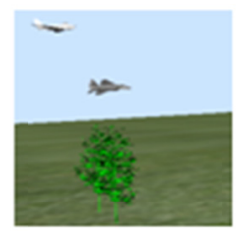

Query:VW27

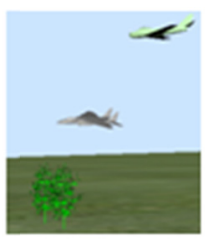

vW28

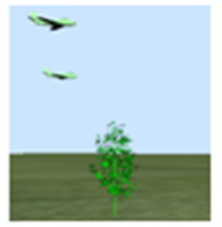

VW20

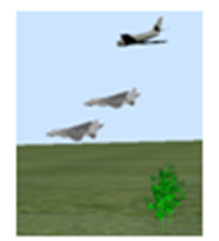

vW29

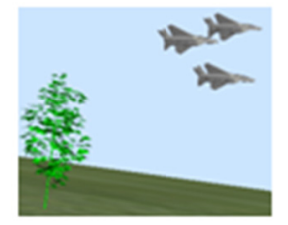

vW21

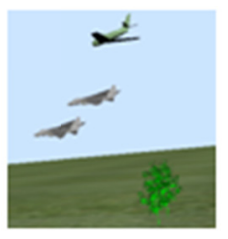

vW30

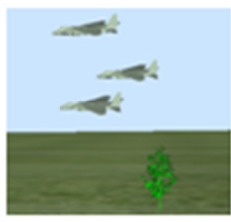

vw22

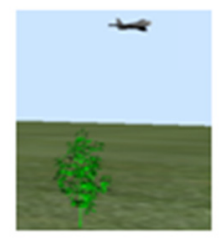

vW18

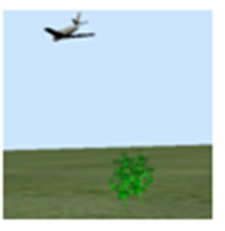

VW26

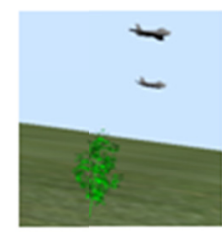

vW19

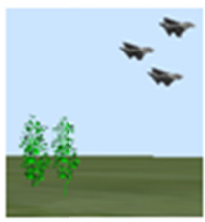

vW31

Figure 14. Returned virtual worlds using the distance D for the virtual world VW27 from our database

\section{Conclusion}

In this paper we proposed an ontology and content based method for retrieving 3D virtual worlds. The proposed ontology scheme that well describes the $3 \mathrm{D}$ virtual worlds could make users more naturally find desired virtual worlds from the proposed 3D virtual world database using the SPARQL query language. The content based method for retrieving virtual world is based on shape of $3 \mathrm{D}$ objects constituting this virtual world. Using the proposed distance, this method shows good results regarding the recall vs. precision plots. It can be used also for matching 2D images with many 2D shapes each one. The proposed methods show good results regarding visual and semantic retrieval for virtual worlds.

\section{References}

Ansary, T. F., Daoudi, M., \& Vandeborre, J. P. (2007). A Bayesian 3d search engine using adaptive views clustering. IEEE Transaction on Multimedia, 9, 78-88. http://dx.doi.org/10.1109/TMM.2006.886359

Chen, D. Y., Ouhyoung, M., Tian, X. P., \& Shen, Y. T. (2003). On visual similarity based 3D model retrieval (pp. 223-232). Eurographics, Granada, Spain.

ENIC search engine. (n.d.). Retrieved from http://www-rech.enic.fr/3dretrieval/

Gong, B., Jianzhuang, L., Xiaogang, W., \& Xiaoou, T. (2011). 3D object retrieval with semantic attributes. In Proceedings of the 19th ACM international conference on Multimedia (MM'11) (pp. 757-758). ACM, New York, NY, USA.

Horrocks, I. (2008). Ontologies and the semantic web. Communications of the ACM, 51(12), 58-67. http://dx.doi.org/10.1145/1409360.1409377

Informatics \& Telematics Institute search engine. (n.d.). Retrieved from http://3d-search.iti.gr/3DSearch

Kassimi, M. A., \& Elbeqqali, O. (2012). Semantic based 3D model retrieval, (ICMCS) (pp.195-199), May 2012.

Lmaati, E. A., Oirrak, A. E., \& Kaddioui, M. N. (2009). A Visual Similarity-Based 3D Search Engine. Data Science Journal, 8, 78-87. http://dx.doi.org/10.2481/dsj.007-069

Lmaati, E. A., Oirrak, A. E., Aboutajdine, D., Daoudi, M., \& Kaddioui, M. N. (2010a). A 3-D Search engine based on Fourier series. Computer Vision and Image Understanding, 114(1), 1-7. http://dx.doi.org/10.1016/j.cviu.2009.09.010 
Lmaati, E. A., Oirrak, A. E., Kaddioui, M. N., Ouahman, A. A., \& Sadgal, M. (2010b). 3D Model Retrieval Based on 3D Discrete Cosine Transform. Int. Arab J. Inf. Technol, 7(3), 264-270.

Lmaati, E. A., Oirrak, A. E., \& Kaddioui, M. N. (2010c). A 3D search engine based on 3D curve analysis. Signal, Image and Video Processing, 4(1), 89-98. http://dx.doi.org/10.1007/s11760-008-0091-2

Niccolucci, F., \& D'Andrea, A. (2006). An ontology for 3D cultural objects. In Proceedings VAST'06 (pp. 203-210), Eurographics Association, Switzerland.

OWL. (n.d.). Web Ontology Language. Retrieved from http://www.w3.org/TR/owl-ref/

Princeton search engine. (n.d.). Retrieved from http://shape.cs.princeton.edu/search.html

Protégé. (n.d.). Retrieved from http://protege.stanford.edu/

RDF. (2004). Resource Description Framework. Retrieved from http://www.w3.org/RDF/

Shilane, P., Kazhdan, M., Min, P., \& Funkhouser, T. (2004). The princeton shape benchmark (pp. 167-178). Shape Modeling International, Washington, DC, USA, June (2004).

SPARQL. (n.d.). Protocol and RDF Query Language, W3C Recommendation. Retrieved January 15, 2008, from http://www.w3.org/TR/rdf-sparql-query/

Tabia, H., Daoudi, M., Vandeborre, J. P., \& Colot, O. (2011). A New 3D-Matching Method of Nonrigid and Partially Similar Models Using Curve Analysis. IEEE Trans. Pattern Anal. Mach. Intell., 33(4), 852-858. http://dx.doi.org/10.1109/TPAMI.2010.202

Tangelder, J. W. H., \& Veltkamp, R. C. (2008). A survey of content based 3D shape retrieval methods. Multimedia Tools Appl., 39(3), 441-471. http://dx.doi.org/10.1007/s11042-007-0181-0

Van Rijsbergen, C. J. (1979). Information Retrieval (2nd ed.). Butterworth-Heinemann.

VRML. (n.d.). Virtual Reality Modeling Language. Retrieved from www.w3.org/MarkUp/VRML/

Xin-ying, W., Tian-yang, L., Sheng-sheng, W., \& Zhengxuan, W. (2008). An Ontology and SWRL Based 3D Model Retrieval System. AIRS, 2008, 335-344.

\section{Copyrights}

Copyright for this article is retained by the author(s), with first publication rights granted to the journal.

This is an open-access article distributed under the terms and conditions of the Creative Commons Attribution license (http://creativecommons.org/licenses/by/3.0/). 